\title{
A Situation Awareness Assistant for Human Deep Space Exploration
}

\author{
Guy A. Boy ${ }^{1,2}$ and Donald Platt ${ }^{1}$ \\ ${ }^{1}$ Human Centered Design Institute, Florida Institute of Technology \\ ${ }^{2}$ NASA Kennedy Space Center \\ \{gboy, dplatt) efit.edu
}

\begin{abstract}
This paper presents the development and testing of a Virtual Camera (VC) system to improve astronaut and mission operations situation awareness while exploring other planetary bodies. In this embodiment, the VC is implemented using a tablet-based computer system to navigate through interactive database application. It is claimed that the advanced interaction media capability of the VC can improve situation awareness as the distribution of human space exploration roles change in deep space exploration. The VC is being developed and tested for usability and capability to improve situation awareness. Work completed thus far as well as what is needed to complete the project will be described. Planned testing will also be described.
\end{abstract}

Keywords: Situation Awareness (SA); Augmented Reality; Human-Computer Interaction (HCI); Tablet Computing; Usability Testing; Space Exploration.

\section{INTRODUCTION}

The Virtual Camera (VC) for human deep space exploration is a virtual assistant that is being developed for use by astronauts and other associated mission operations personnel as they use human-piloted rovers to explore the surface of other planets [1]. The VC concept is based on incremental upgrading of a suboptimal 3-D geographical and geological database. It is an interactive window on the world as we know it at the time it is being used. Such an interactive window enables the user to maintain better situational awareness, to navigate and further explore space. It can be used for training, data analysis and augmentation of actual surface exploration.

The VC for human space exploration was originally described for use by astronauts navigating a surface exploration rover on a remote body such as the Moon [1]. Further analysis has indicated that such an interactive window to data in multiple dimensions has many other applications in domains such as aviation, medicine, control systems and finance. This paper presents a tablet version of the VC for deep space exploration using a scenario-based design approach. This work brings to human space exploration

adfa, p. 1, 2011

(C) Springer-Verlag Berlin Heidelberg 2011 
the use of advanced interaction techniques not previously used in spacecraft cockpit systems.

\section{MOTIVATION}

There are significant challenges for astronauts while operating in the space environment, including increased levels of stress and workload, combined with decreased situation awareness. Astronauts and their exploration are evolving in an environment that is characterized by progressive discovery.

Human deep space missions will require increased roles for astronauts on-board as well as increased on-board automation. Previous work has determined that robots and humans need to work together in co-coordinated efforts [2]. It is envisioned that the VC can integrate data from both human-piloted and autonomous robotic explorers to improve the safe and efficient human exploration of a deep-space location (i.e., Moon, asteroid or Mars). On-board software and displays will integrate and manage all relevant systems as well as mission data. This information will include system health and status, caution and warning, traverse execution and mission timeline parameters.

There will be a need to make decisions based upon the collection and analysis of raw data to provide predictive information [3]. This information needs to be presented to crews in a way that enhances situation awareness. One way to do this is with new interactive environments such as 3-D tablet computer systems using advanced interaction techniques based upon accelerometer- and gesture-based inputs.

\section{RELATED WORK}

Interactive cockpits, context-sensitive systems and remote agent knowledge representation are being developed for a number of domains including aviation [4], nuclear power plants [5] and even passenger car driving [6]. The VC will demonstrate these requirements in a system usable for deep space human exploration.

In early tests using a human-piloted rover in space-simulated environments it became clear that astronaut situation awareness needed to be improved [7]. Often when operating in environments such as the NASA Desert Research And Technology Studies (RATS) space exploration test bed, rover operators needs people outside the rover vehicle to guide for safe operation of the vehicle. This will not be possible for all rover or other space vehicle operations in deep space.

\section{DESIGN GOALS}

In deep space, situation awareness will be difficult due to the lack of input from the ground that astronauts are typically used to as well as the skill-retention concerns and environmental isolation from the environment under exploration. 
The $\mathrm{VC}$ is playing the role of a remote agent for mission operations personnel and scientists. Functions that were previously done by human experts on the Earth for missions near Earth will be transferred to the on-board VC. With this transfer there will be emergent behaviors, which will be explored and identified through this research.

NASA is currently developing technology for use with deep space surface exploration. A centerpiece of this exploration effort is the Multi-Mission Space Exploration Vehicle (MMSEV). This vehicle is being designed for use by astronauts on a variety of missions including Near-Earth Asteroid exploration. It is anticipated that the VC would support exploration conducted by this vehicle. Figure 1 shows a MMSEV concept that is based upon the Surface Exploration Vehicle that has been tested at the NASA Desert RATS analog test-bed. Part of the goal of designing a VC for space exploration is to afford the most natural and intuitive human-system interaction. A tablet computer interface provides a small, portable platform with a very common user interface that is gaining wide acceptance.

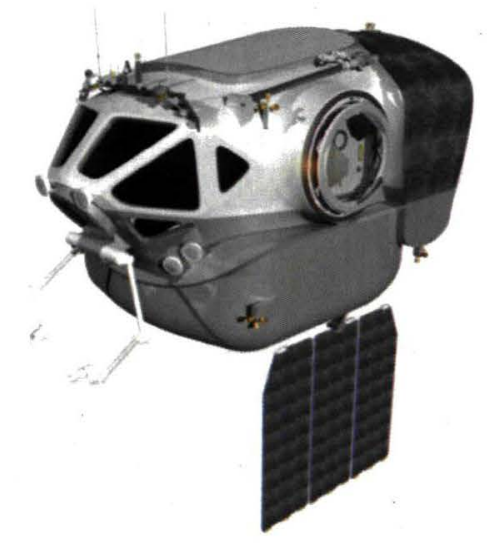

Fig. 1. Multi-Mission Space Exploration Vehicle (MMSEV) for Astronaut Deep Space Exploration (NASA).

A tablet allows gestures rotational interaction that astronauts have expressed that would be useful in the space environment. Two tablets are being used by Russian cosmonauts for recreation on the International Space Station [8], and by pilots for the use of electronic charts in commercial aircraft cockpits [9]. 


\section{WORK COMPLETED}

A human-centered design approach is being used to develop the tablet-based VC. The first step was to develop low-level prototypes to capture the basic design requirements for the VC. These were then presented to potential users during the fall of 2011 at the NASA Desert RATS test sessions. Horizontal prototypes were developed that captured the basic interaction requirements for the VC and were used to demonstrate an operational scenario. Currently, a complete vertical prototype is being developed with all of the interaction capability using a tablet PC device.

\subsection{User Requirements Elicitation}

The authors conducted a survey of potential users and stakeholders (astronauts, mission operations personnel, and scientists) for the VC system at the 2011 NASA Desert RATS analog exploration testbed. The main questions during potential user interviews involved their background, general system uses, interface type and data display parameter formats desired.

User feedback indicated a device that provides real-time sensor knowledge display combined with multi-modal information display (vision and auditory for instance) would be useful. They also suggested that the VC should help in the navigation process and also display information about consumables such as power level, fuel levels and life support systems. This information was used to develop initial horizontal prototypes showing possible uses, screens and interactions for the VC tablet device.

Use cases for the VC were defined, and then a horizontal prototype was developed. This allows human-in-the-loop simulations to take place where the system can be tested with a variety of users. Other scenarios can also be illustrated in this way. Users can interact with actual system prototypes, which are being developed on the tablet-based computer system that will be used for the end product. This then allows the full functionality to be incorporated after initial user testing and feedback.

The horizontal prototype screens show the functionality and capabilities of the VC although the database interaction was not yet be possible. This horizontal prototyping allows astronaut, scientist and mission operations personnel to provide feedback as to the usefulness of the screens being developed.

Asteroid Itokawa has been modeled using a stereo lithography database format (STL) created by Gaskell [10] based on data from the Japanese asteroid mission Hayabusa. This data was used in the developed prototypes to simulate flying close to asteroid Itokawa and the resulting VC interactions.

The VC interface screen in Figure 2 shows the actual asteroid surface as projected by the STL file. This is how an astronaut would interface with the VC as they approached the asteroid in an MMSEV. The vehicle's velocity in $\mathrm{X}, \mathrm{Y}$ and $\mathrm{Z}$ coordinated is shown in the upper left. Next to the actual velocity are bars of relative velocity. The color green represents a safe speed for the operating environment. If the velocity was slightly high, then the bar would turn yellow and if it was a dangerous level it would turn red. The velocity vector is simulated by the green arrow, which also points in the basic direction of travel. 


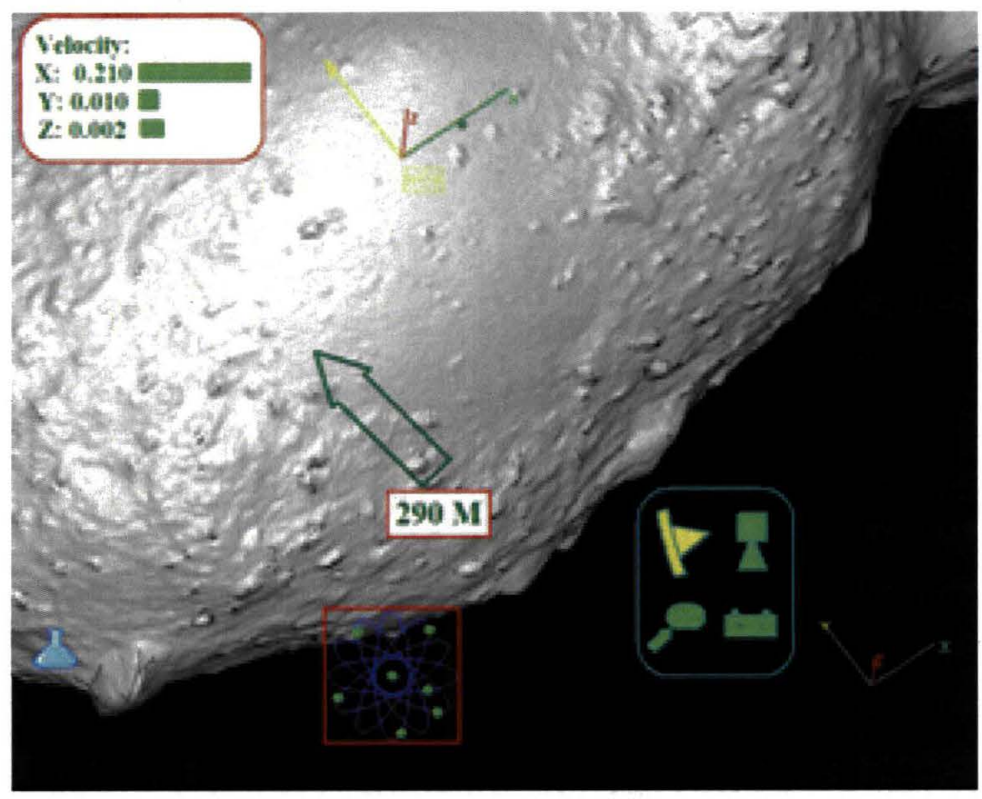

Fig. 2. The VC Horizontal Prototype Interface.

An information cartouche in the lower right shows icons for communication link, thruster systems in use, electrical power and collision potential going clockwise from the upper left. Other information elements include a radiation sensor-warning icon in the lower middle indicating an on-board radiation sensor has detected radiation. In the lower left the blue beaker icon indicates an area of scientific interest.

In this example scenario the astronaut can determine to fly toward the beaker icon to attain a mission science goal or ignore the icon and fly in another direction. If another direction is chosen, other areas of interest could be displayed by icons. Sensor data is also compared to the current vehicle configuration and operational mode to determine possible safety concerns and out of nominal operation.

Figure 3 shows some of the safety information displays possible in the VC. In this case the astronaut is flying closer to the asteroid and is close to a mountain feature on the surface. Dust may be kicked up as he approaches and a dust warning has been triggered. The fuel level in the thruster triad currently active has reached a threshold that has triggered the rocket icon to turn red. Also the communications link with either the Earth or a nearby habitation system has deteriorated causing the communications (antenna) icon to turn yellow.

As the vehicle approaches the area of interest (in either a simulation mode or later in actual deep space exploration) the beaker icon changes to surface colors to indicate the exact area for exploration. This allows the astronaut to determine where to aim 
high resolution sensors, take high resolution images or to collect samples. Similarly mission operations personnel or scientists could "fly" mission scenarios and determine plans for exploration sorties at the actual asteroid. Annotations and other information screens can also be displayed as required when the exploration context calls for them. Examples could be radiation sensor alarms, loss of communication, and impending contact with the asteroid surface or a crater on the moon. Colors could represent either physical concentrations or an exploration priority scheme.

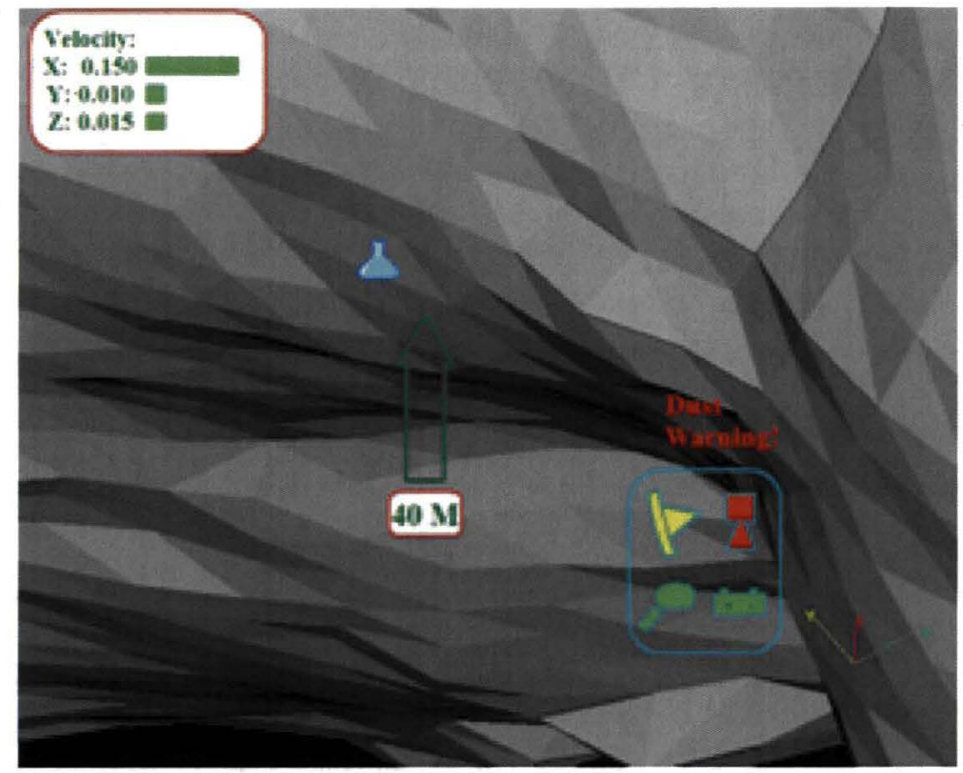

Fig. 3. VC Prototype Screen Showing Warnings

These screens can be put together into a storyboard as the astronaut in the simulated MMSEV flies closer to and around the asteroid surface. This allows a movie to be created that simulates a mission scenario. Other types of information displays possibilities with the VC interaction include coloring the surface to correspond with certain areas of scientific or exploration interest. The elevation information would still be visible but additional information about areas that may potentially be explored could also be represented by different colors.

Emergent uses of the system and behaviors enabled or limited by the VC are discovered by the use of prototypes. A vertical prototype with the interaction functionality afforded by a tablet computer is now under development. This will allow complete testing of interactivity of the system. Figure 5 shows a screen from the VC vertical prototype using the Google Maps database. The information cartouche is displayed with the collision icon active if the user zooms in beyond a settable level. The direction arrow shows the direction of the accelerometer interaction sensor. The upper left shows where the VC application started as a central empty blue circle (in terms of geographic position) as well as a second red dot indicating the current view position 
in relation. Scale is also user settable. Scientific (beaker), resource (water drop) and user annotations (information) icons are also present.

\section{FUTURE WORK}

The final product of this work will be a tablet-based prototype VC device using the unique interaction capabilities of the tablet platform. These include portability, accelerometer-based 3-D interaction and the ability to be submerged into the 3-D environment. The interaction features such as icons, colors and annotations described are being incorporated.

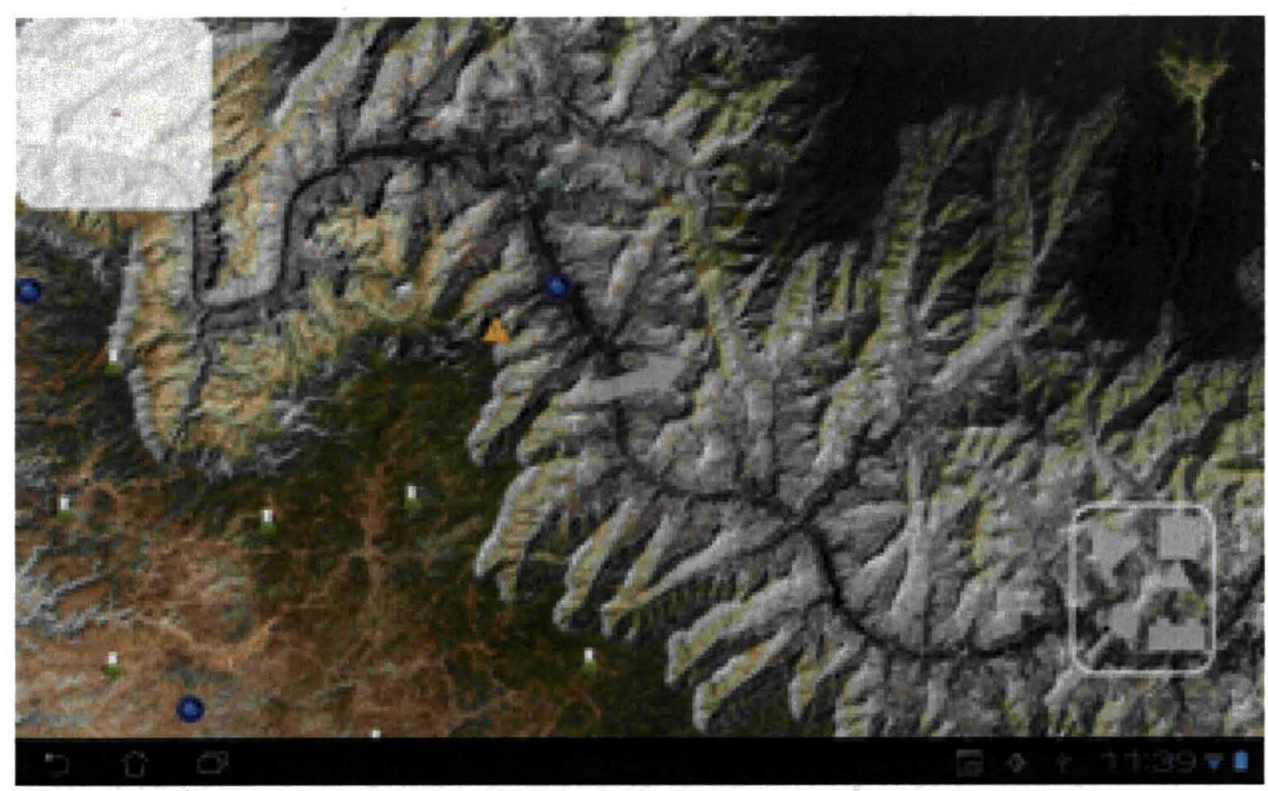

Fig. 4. The VC Vertical Prototype Interface Using Google Maps as the Database.

This VC device will form the basis for evaluating the utility of the interactive database concept for improving situation awareness in the unique environment of deepspace human-piloted space exploration. Both mission planners and astronauts can conduct a simulated traverse using the $\mathrm{VC}$, annotating areas of exploration interest or safety concern prior to conducting the actual traverse in the field. The tablet GPS location system can then be used to assist the astronauts as they follow through the traverse at a terrestrial space exploration analog site such as NASA Desert RATS.

\section{CONCLUSION}

The virtual camera for planetary exploration will assist in future exploration involving humans and robots. It is designed to provide improved situational awareness and 
augmented reality to define areas of interest and safety concern on remote planetary bodies. It will act as remote agents for mission planners and scientists capturing their knowledge and expertise as astronauts explore the solar system. It is claimed that the advanced interaction media capability of the VC can improve situation awareness as the distribution of human space exploration roles change in deep space exploration.

Human-centered-design techniques are being applied to the development of this tool involving all potential user groups from the beginning of the design process. Moving forward, the VC prototype will be finalized and further testing with domain experts will take place. Changes found in this testing will be incorporated into an updated VC and final evaluation will take place comparing this system to other interaction devices currently being developed. Recommendations will be made for future work to create a flight-ready VC capability. The advanced interaction possibilities with the VC will allow new types of displays and an immersive environment to assist astronauts to comprehend and project into the future situation awareness as their autonomy from ground control resources increases in deep space.

\section{ACKNOWLEDGMENTS}

We would like to thank Ph.D. students, Kara Schmitt and Lucas Stephane for their advice and support. NASA Kennedy Space Center's Rebecca Mazzone and Michael Conroy have provided logistical support for this project. Darrell Boyer and Matthew Long have provided software development support for this project.

\section{REFERENCES}

1. Boy, G.A. et al: The Virtual Camera: A Third Person View, Third International Conference on Applied Human Factors and Ergonomics (2010)

2. Deans, .M.C. et al,.: Field Testing Next-Generation Ground Data Systems for Future Missions, 42nd Lunar and Planetary Science Conference (2011)

3. Cummings, M. et al: Past, present and future implications of human supervisory control in space missions, Acta Astronautica, 62, 648 - 655 (2008)

4. Harper, R, Hughes, J.A. \& Shapiro, D.: Harmonious Working and CSCW: Computer Technology and Air Traffic Control. In: Bowers, J. and Benford, S. (eds.) Studies of Computer Supported Cooperative Work. Amsterdam, North-Holland: s.n. (1991)

5. US Nuclear Regulatory Commission: Technical Basis and Implementation Guidelines for A Technique for Human Event Analysis (ATHEANA), United States Government, Washington, D.C., NUREG-1624, Rev. 1 (2000)

6. Raja, A.K, et al.: Power Plant Engineering. New Age International (2006)

7. Boy, G.A.: The Virtual Camera Progress Report, report to NASA (2011)

8. Malik, T.: iPads Would Be Great in Space, Astronaut Says, http://www.space.com/14822ipads-space-station-astronaut.html (2012)

9. Paur, J.: FAA approves iPads for pilots' electronic charts. Wired, Condé Nast, New York, Feb 28 (2011) 
10. Gaskell, R. et al.: Landmark Navigation Studies and Target Characterization in the Hayabusa Encounter with Itokawa, AIAA 2006-6660, AAS/AIAA Astrodynamics Specialists Conf., Keystone, CO, (2006) 